\title{
AN ANALYSIS REGARDING THE POSSIBILITY OF USING FUZZY LOGIC IN INVENTORY MANAGEMENT
}

\author{
CLAUDIU- LEONARDO STOIA \\ Department of Engineering and Management, Lucian Blaga University of Sibiu, Romania, \\ claudiu.stoia@ulbsibiu.ro
}

\begin{abstract}
The paper presents a brief state-of-the-art survey regarding the use of fuzzy logic in inventory management. Its goal is to motivate enthusiastic entrepreneurs to take into account the benefits of using fuzzy logic inventory control systems. It offers a guide to model an inventory system having a free fuzzy tool as starting point.
\end{abstract}

Keywords: fuzzy, control, inventory, guide

\section{Introduction :}

An inventory system represents the set of policies that supervise and control the levels of inventory and decide what levels should be kept, when to order and how much to order.

Inventory control is needed to make good economic sense, balancing between supply and demand (Riadi, 2010). Inventory must be enough to meet the demand and be cost effective (Petrovic and Duenas, 2006).

There are three main types of inventory systems : a continuous inventory system (fixed order quantity), a periodic one (fixed time period : weekly or monthly) and an inventory system that combines the first two types (Bălan, 2006). In a periodic review model one may place an order only at specific time period while, in a continuous one- an order is placed anytime. But, whatever the inventory management policy, one must make the following decisions in each period (Pope, 1981, p.265):

- Whether or not to order;

- How much to order;

- How much to expedite.

"' The decision-making and the exercise of will are very strenuous, especially if they are made in a hurry and without the subconscious' help" '(Bertrand Russel). Let's assume that manager subconscious' role is played by a computer program.

So it begins the idea of Artificial Intelligence (AI) and its branches (i.e. expert systems, artificial neural networks, genetic algorithms and fuzzy systems).

The fuzzy approach in decision-making started in 1970, when Bellman and Zadeh have presented an article called 'Decision-making in a fuzzy environment'. In the early 1970s, Mamdani has managed to control a steam engine plus boiler's system using tens linguistic rules and the fuzzy sets.

In order to maintain and improve a complex system, one needs the proper understanding of differential equations, which model conventional control techniques. This is pure science (and also a huge source of distress).

On the other hand, companies rely on key-employees who use their intuition combining data and control strategies that cannot be captured into unique analytic control law. This is art.

Does the science meet the art, in a modern company? Yes, it does, in the A.I. field. Knowledge-based (expert) systems have the purpose to capture the métis (old greek word for skill, craft, wisdom) available from key-operators. The Fuzzy rule-based control is an expert system that contains fuzzy IF [present conditions]-THEN [action] rules. Fuzzy logic simulates the nature of human decision processes .This is a clearly improvement over methods based on binary logic which are widely used in industrial controllers (Babuska and Mamdani, 2008).

Fuzzy logic is a form of multi-valued logic introduced in 1930 by Jan Lukasiewicz (known also for Lukasiewicz-Moisil algebras). Fuzzy logic regards fuzziness or vagueness. In the narrow sense fuzzy 
logic deals with the representation of knowledge and reasoning or inference. It is a branch of fuzzy sets theory developed by Lotfi Zadeh in 1965.

According to Zadeh, fuzzy sets are "classes" with a continuum of grades of membership, such as "the class of beautiful women" or "the class of tall men". These classes play an important role in human thinking, despite of their vague definition. A fuzzy set A in a space of points X is defined by a membership function which associates each point in $X$ with a real number in the $[0,1]$ interval.

The Zadeh's idea of using Fuzzy Control algorithms is based on introducing the knowledge base into the controller, such that his output is determined by the rules captured from the expert (Aguilar et al. 2012).

The paper emphasizes the remarkable capacity of fuzzy if-then rules modelling language to bypass the need for bivalent-logic-based models (Zadeh, 2008, p.2776) in inventory management applications.

Figure 1: Fuzzy control system

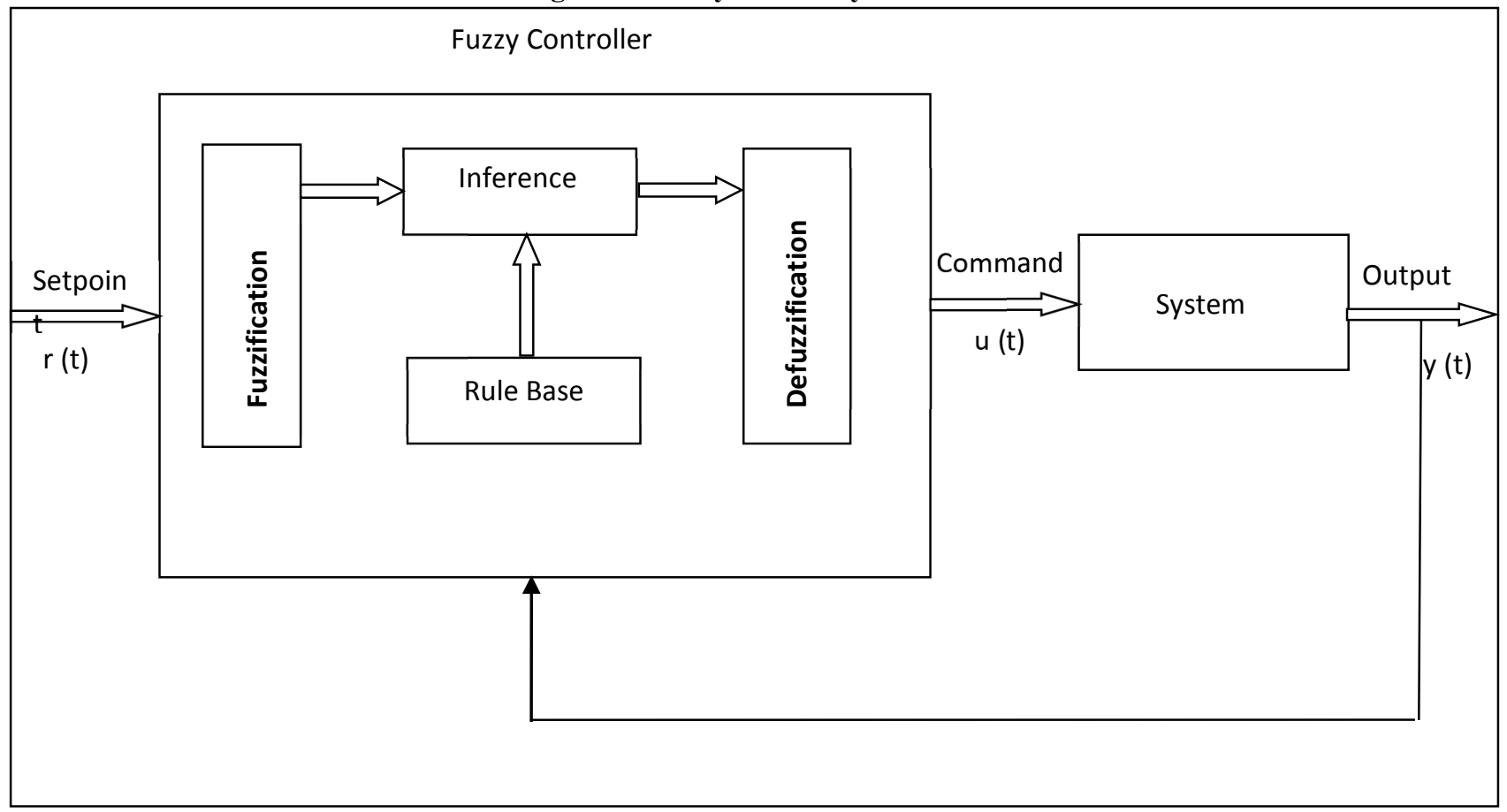

(Source : Aguilar et al. 2012, p.382)

\section{State-of-the-Art}

Tanthatemee and Phruksaphanrat (2012) have realized a brief state- of-the- art review regarding the use of Fuzzy Logic Control. They found applications regarding scheduling and system controls including inventory control (Petrovic and Duenas, 2006 apud Tanthatemee and Phruksaphanrat, 2012). There is, also, a fuzzy simulation model of a single item inventory system with variable demand that uses Fuzzy Logic Control, presented by Yimer and Demirli in 2004.

The review's authors conclude that there are few researchers (Babai and Dallery, 2006 or Kamal and Sculfort, 2007) who take into account both uncertainty quantities of demand and supply.

Therefore, Tanthateme and Phruksaphanrat, propose Fuzzy Logic Control to treat the uncertainty concerning demand and supply in inventory control system (a continuous one, simulated with MATLAB's Fuzzy Toolbox). The obtained results clearly show that their fuzzy model can extremely save the total inventory cost.

Khanlarpour et al., 2013 have developed a hybrid model for a diary environment. First, they have specified the next variables : weather conditions, status of rival companies, customer income status, religious and secular status, effect of holidays and the tourism and customer satisfaction as main influential factors of dairy products' consuming. Using fuzzy logic, they have specified the optimal order point. On the other hand, by applying genetic algorithms, they have modeled the optimal order quantity.

\section{Short guide to model an inventory system with a free available fuzzy tool}

First step is to pick-up a convenient fuzzy tool.

Ederson and his collaborators presented in 2010 an analysis of ten fuzzy tools available on the market. These tools help businesses in developing fuzzy applications. 
Table 1: Comparative about available tools for fuzzy systems

\begin{tabular}{|c|c|c|}
\hline $\begin{array}{c}\text { Fuzzy } \\
\text { Software }\end{array}$ & Advantages & Disadvantages \\
\hline $\begin{array}{l}\text { MatLab’s } \\
\text { Fuzzy Logic } \\
\text { Toolbox }\end{array}$ & $\begin{array}{l}\text { Large number of resources } \\
\text { Suitable graphic interface } \\
\text { Simplicity in the construction of fuzzy systems }\end{array}$ & Paid tool \\
\hline FuzzyTech & $\begin{array}{l}\text { Large number of resources } \\
\text { Suitable graphic interface } \\
\text { Project report routine } \\
\text { Export of source code }\end{array}$ & Paid tool \\
\hline XFuzzy & $\begin{array}{l}\text { Free tool } \\
\text { Documentation } \\
\text { Open source code allows new implementations } \\
\text { Input rules easy to create } \\
\text { Inference models of Mamdani and } \\
\quad \text { Takagi-Sugeno }\end{array}$ & $\begin{array}{l}\text { Do not generate reports of project } \\
\text { implemented }\end{array}$ \\
\hline
\end{tabular}

(Source : Table processed after the conclusions of Ederson et al., 2010, p.290)

According to Aguilar et al., 2012, the procedure for implementing fuzzy techniques to control systems consists of two stages :

I. the first stage, before the control algorithm's execution, includes :

- establish the linguistic variables for the controller's inputs and outputs;

- defining each variable's fuzzy set;

- defining the sets' membership functions;

- establishing the rule base;

- defining the fuzzification, inference and defuzzification mechanism.

II. the second phase, to be finished with each step of the control algorithm, contains :

- obtaining the accurate input values;

- fuzzification : assigning the accurate values to the fuzzy input sets and calculating the degree of membership for each set;

- inference or reasoning;

- defuzzification procedure: obtaining the accurate output values from the inferred fuzzy sets. These accurate values are the commands applied to the system in order to be controlled.

In order to illustrate a fuzzy system one may use a fuzzy tool that benefits from simple and elegant graphic interface, like ' qtfuzzy'" (Rada-Vilela, 2013).

The two ready to hand input variables of an inventory control system are demand and availability of supply. Demand may have the degrees : low, medium and high, while the availability of supply may be low and high, for the simplification. The output variables could be order quantity with the degrees : low, medium, high, and reorder point with the terms : low, average, high and very high.

Figure 2: Establishing the linguistic variables for the controller's inputs and outputs

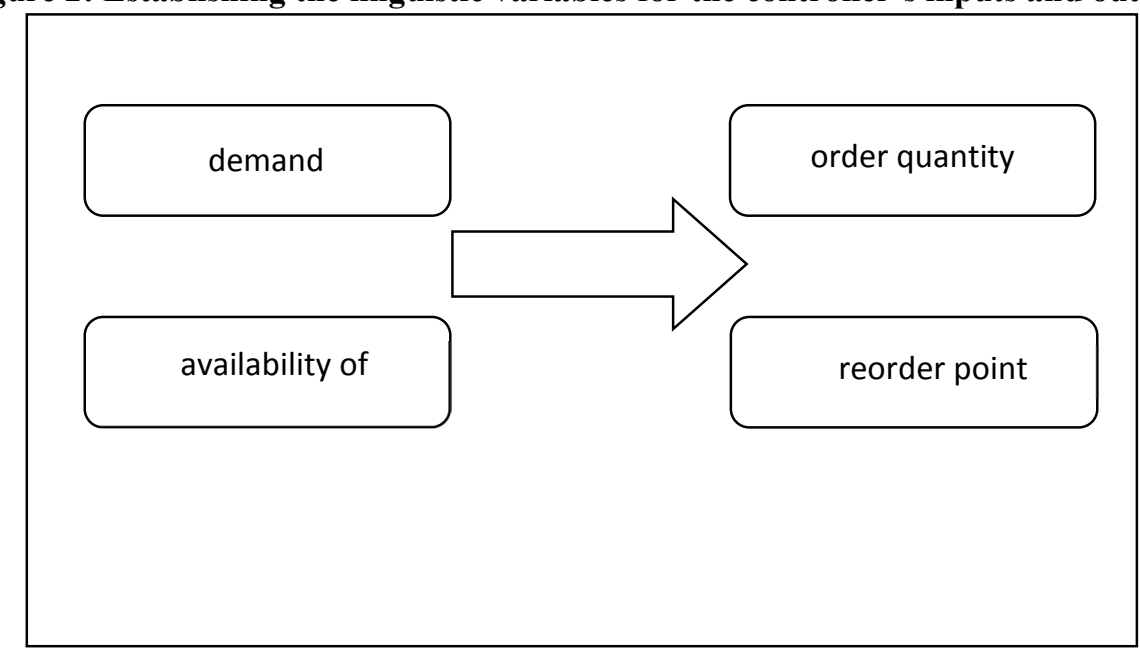

Figure 3: Defining each variable's fuzzy set 


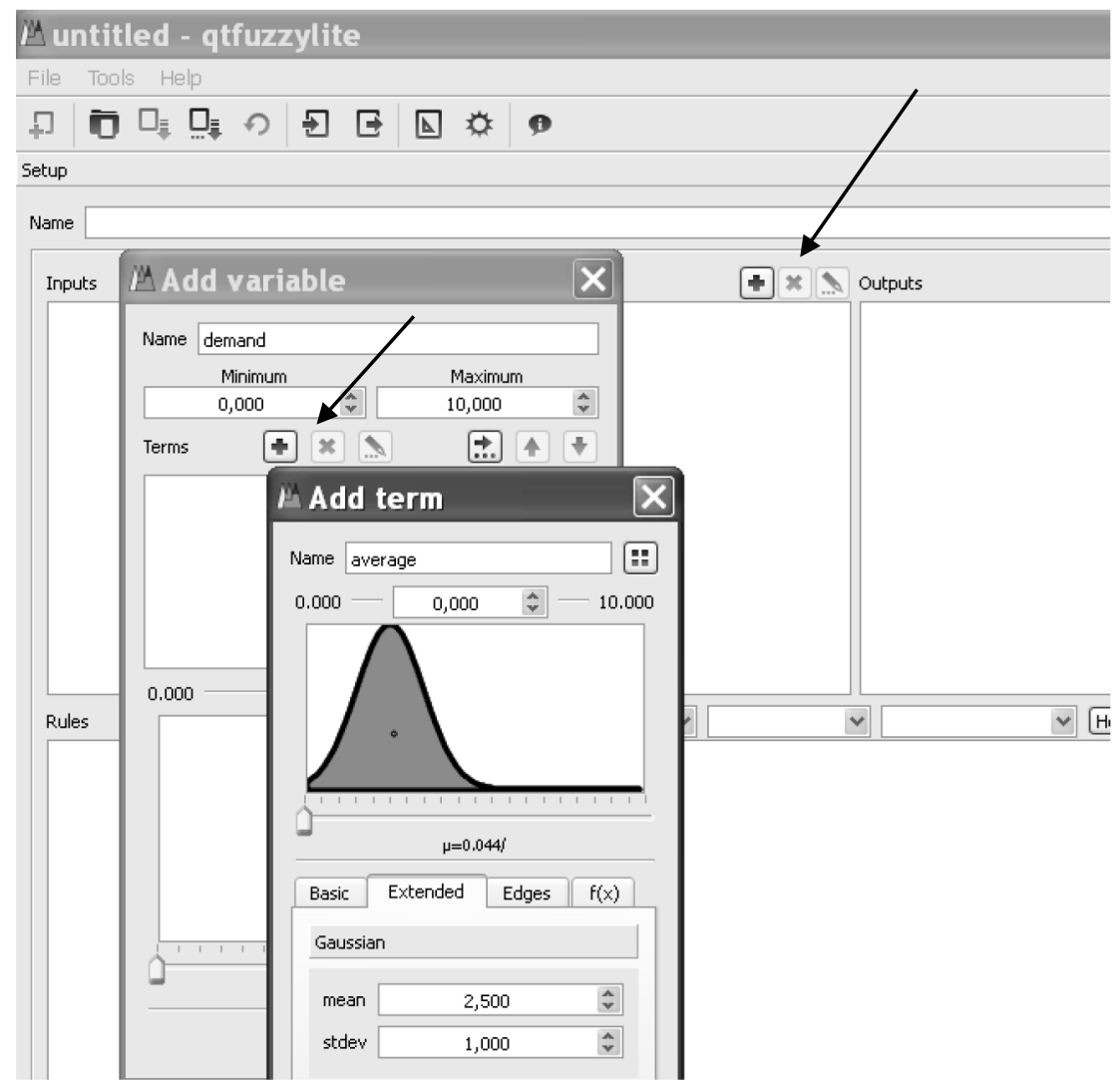

Source : (Fuzzylite 3.1-win 32, available at : www.fuzzylite.com)

Figure 4: Establishing the rule base

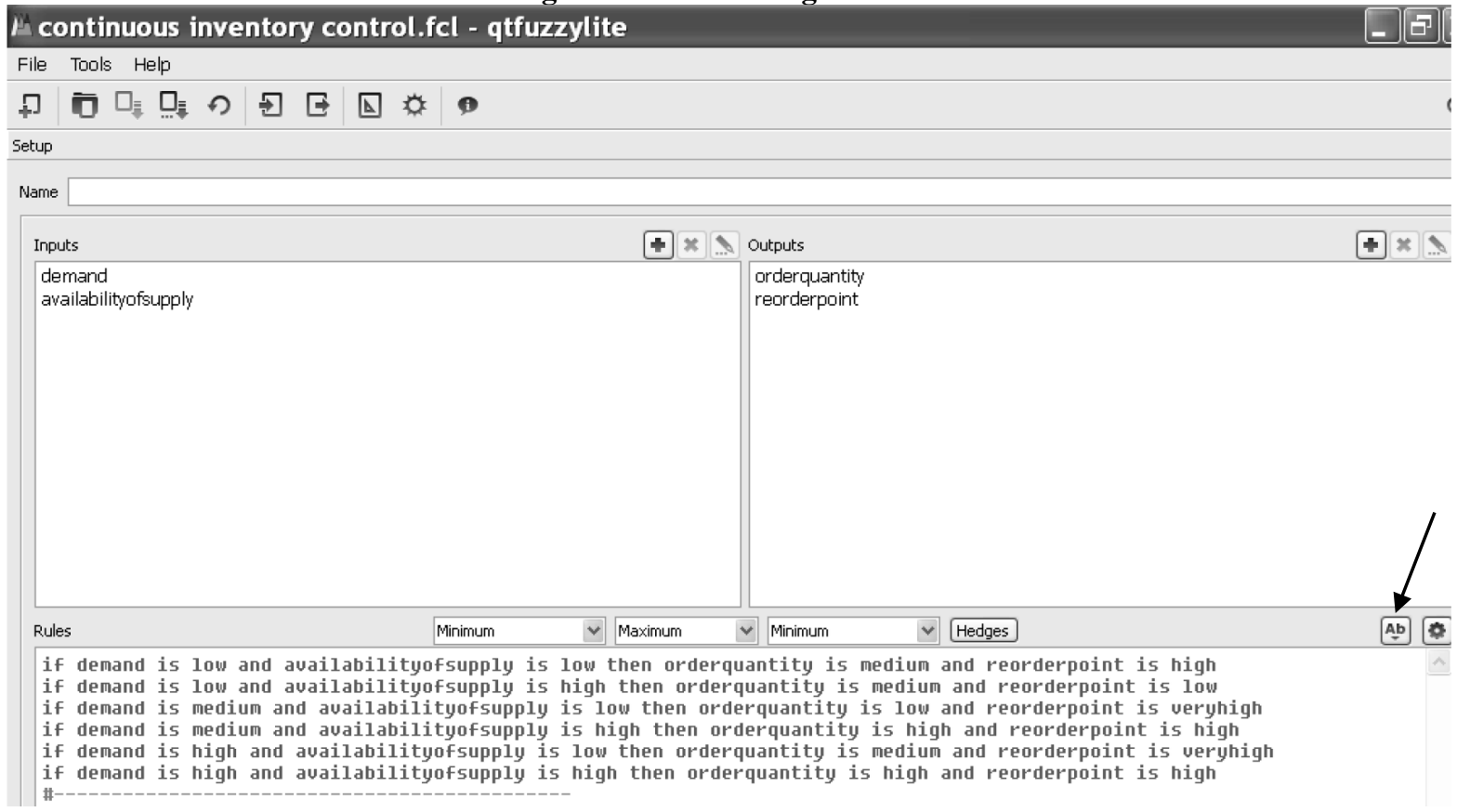

Source : (Fuzzylite 3.1-win 32, available at : www.fuzzylite.com) 
Figure 5: Demonstration of a Fuzzy rule-based system

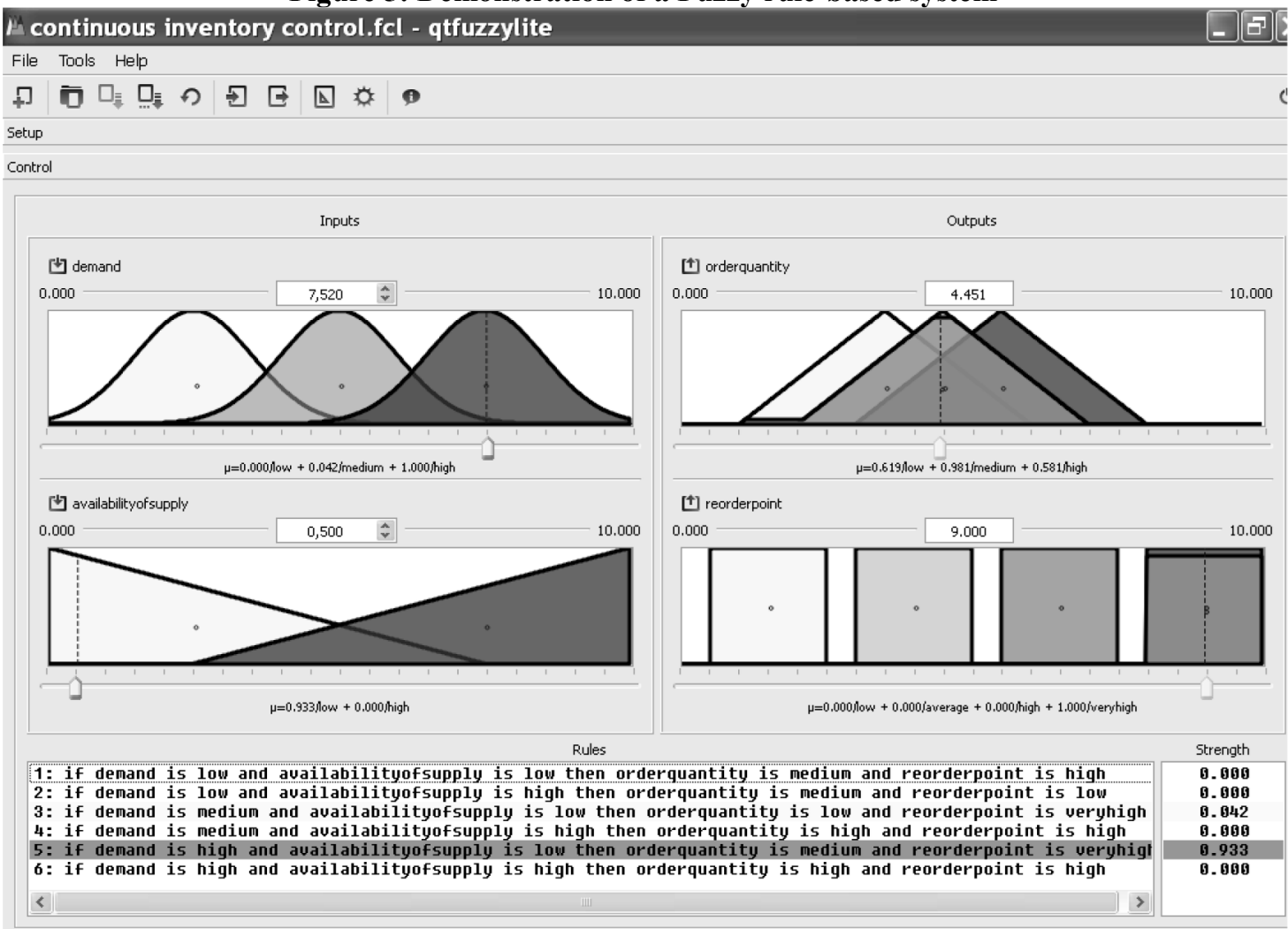

Source : (Fuzzylite 3.1-win 32, available at : www.fuzzylite.com)

\section{Conclusion}

The best way to get started with a Fuzzy Inventory Control system is to select a free high-potential application, like XFuzzy. Start small, and grow the system. As the company becomes more experienced, the application can be developed adding more knowledge.

The use of Fuzzy Logic in inventory management field provides strategic benefits like:

- an elegant and effective tool for inventory decision-making;

- savings of total inventory cost;

- more precise mimic of systems' behaviour, due to linguistic rules which are converted in their mathematical equivalents by fuzzy system (in comparison with conventional way, i.e. the use of differential equations);

- system's flexibility in comparison with a stochastic model, thanks to possibility of continuously reevaluation of both order quantity and reorder point (Tanthatemee and Phruksaphanrat 2012) ;

- back-up for an key-expert and therefore a training tool for novice employees;

\section{Future work}

The machinery of linguistic variables and fuzzy if-then rules plays a pivotal role in the conception and design of control systems (Zadeh, 2008, p.2769). Its applicability in inventory management field is far from being exhausted.

The rise trend is to combine fuzzy logic with artificial neural networks or with genetic algorithms in order to enable some forms of learning. These are the cooperative models of hybrid systems.

\section{References}

- Aguilar, R.M.; Muñoz, V.; Callero, Y. (2012) Control Application Using Fuzzy Logic: Design of a Fuzzy Temperature Controller, Fuzzy Inference System - Theory and Applications, Dr. Mohammad Fazle Azeem (Ed.), ISBN: 978-953-51-0525-1, InTech, pp.379-396, Available from: $\mathrm{http} / / / \mathrm{www}$.intechopen.com/books/fuzzy-inferencesystem-theory-and-applications/controlapplication-using-fuzzy-logic-design-of-a-fuzzy-temperature-controller

- Babuska, R.; Mamdani, E. (2008) Fuzzy control, Scholarpedia, 3 (2) :2103;

- Bălan, C. (2006) Logistica, Bucureşti : Uranus, p.191; 
- Ederson, L.P.; Luis Felipe, S.; Rejane, F.; Rolf, F.M (2010) Qualitative Study Of Software for Fuzzy System Simulation and Development, Recent Advances in Neural Networks, Fuzzy Systems \& Evolutionary Computing,

Available at http://www.wseas.us/e-library/conferences/2010/Iasi/NNECFS/NNECFS-43.pdf

- Khanlarpour, E.; Fazlollahtabar, H.; Mahdavi, I. (2013) Designing an Intelligent Warehouse Based on Genetic Algoritm and Fuzzy Logic for Determining Reorder Point and Order Quantity, Computer Science and Information Technology 1(1): 1-8, Available at: http://www.hrpub.org/download/201307/csit.2013.010101.pdf

- Petrovic, D.; Duenas, A. (2006) A fuzzy logic based production scheduling/rescheduling in the presence of uncertain disruptions, Fuzzy Sets and System, vol.157, 2273-2285;

- Pope, J.A. (1981) An Inventory Management Simulation, Journal of Experiential Learning and Simulation 3-4, pp.261-269;

- Rada-Vilela, J. (2013) Tutorial on qtfuzzy, accessed at 11.10.2013, Available at : https://www.youtube.com/watch?v=8UQghVz8N9A

- Riadi, A. (2010) A study on fuzzy inventory control system under demand uncertainty, Master's thesis, Tokyo University of Marine Science and Technology;

- Tanthatemee, T.; Phruksaphanrat, B. (2012) Fuzzy Inventory Control System for Uncertain Demand and Supply, Proceedings of the International MultiConference of Engineers and Computer Scientists 2012, Vol.II, IMECS 2012, March 14-16, Hong Kong ;

- Yimer, A.D.; Demirli, K. (2004) Fuzzy modeling and simulation of single item inventory system with variable demand, IEEE annu. Meeting of the North American Fuzzy Information Processing Society Banff, vol.2, Alberta, Canada, pp.985-989;

- Zadeh, L. (1965) Fuzzy sets, Information and Control, Vol.8, pp.338-353;

- Zadeh, L. (2008) Is there a need for fuzzy logic? , Information Sciences 178, pp.2751-2779;

- http://code.google.com/p/fuzzylite/downloads/detail?name=fuzzylite-3.1-win32.zip, accessed : 11.10.2013; 